\title{
Synthesis and Properties of Ionic Liquids: Imidazolium Tetrafluoroborates with Unsaturated Side Chains
}

\author{
Gwan-Hong Min, Taeeun Yim, Hyun Yeong Lee, Dal Ho Huh, Eunjoo Lee, \\ Junyoung Mun, Seung M. Oh, and Young Gyu Kim* \\ School of Chemical and Biological Engineering, Center for Energy Conversion \& Storage, Seoul National University, \\ Seoul 151-744, Korea. "E-mail: seungoh@plaza.snu.ac.kr;ygkim@snu.ac.kr \\ Received January 27, 2006
}

\begin{abstract}
Imidazolium tetrafluoroborate ionic liquids having unsaturated aliphatic side chains were synthesized and characterized. Most of them are liquid at room temperature and all of them are stable up to $300{ }^{\circ} \mathrm{C}$. Some imidazolium tetrafluoroborates with an allylic side chain showed much wider voltage windows on the platinum electrode, better conductivities, and lower viscosities compared with the corresponding ionic liquids containing the saturated side chains.
\end{abstract}

Key Words : Ionic liquids, Imidazolium tetrafluoroborates, Unsaturated side chains, Synthesis, Physicochemical properties

\section{Introduction}

Ionic liquids (ILs) have been investigated as an ion conductive matrix ${ }^{1}$ as well as a reaction solvent ${ }^{2}$ due to their unique physical properties; a wide liquid range, high ionic conductivity, a wide voltage window and non-volatility. ${ }^{3}$ Therefore, ILs have attracted much attention as a promising alternative to the conventional organic electrolyte and the organic solvent systems.

The problems of early developed ILs with aluminum chloride anions were their corrosiveness and toxicity. After Wilkes and Zaworotko reported the air and water stable ILs with a tetrafluoroborate anion in $1992,{ }^{4}$ ILs have attracted much attention as a potential candidate for use in advanced electrochemical applications. ${ }^{5}$ Typical ILs are composed of nitrogen-containing cations coupled with halides or polyatomic anions. The representative cations are imidazoliums, pyridiniums, and quaternary ammoniums. The anions are usually halides, $\mathrm{BF}_{4}{ }^{-}, \mathrm{PF}_{6}{ }^{-}$and $\mathrm{N}\left(\mathrm{SO}_{2} \mathrm{CF}_{3}\right)_{2}{ }^{-}$. Among these, the ILs with imidazolium cations exhibit higher ionic conductivities and lower viscosities. ${ }^{6}$ Lots of investigations on imidazolium-based ILs for capacitors, ${ }^{5 b, 7}$ solar cells, ${ }^{8}$ fuel cells $^{5 b, 9}$ and batteries ${ }^{5,10}$ have been carried out. Among the anions, the tetrafluoroborate anion is also widely used due to its competitive properties and low cost.

Several studies have been done to control physical properties of ILs through the design of their cations and anions for practical use. The substitution of alkyl chains on the imidazolium ring and the selection of large anion species have been the main means to control the properties of imidazolium-based ILs. However, it was difficult to modify the properties merely by changing the length of alkyl chains without increasing the melting point of the salts. ${ }^{3,6}$

To overcome this problem, a novel design is required to obtain the desired properties of ILs. The ILs with novel anions such as unsymmetrical borates seem to be an efficient way to achieve the goal while the problem of their high costs still remains. ${ }^{11}$ The ILs with functionalized side chains could well be a solution and the task-specific ILs can be representative examples. ${ }^{12}$ We have prepared several new imidazolium tetrafluoroborate ILs with unsaturated side chains on the imidazolium ring and report their physical and electrochemical properties as follows. ${ }^{13}$ Among them, those with an allylic side chain showed interesting physicochemical properties. Although a few imidazolium tetrafluoroborate ILs with an allyl side chain have been synthesized recently, no systematic work including characterization of their physicochemical properties has been done for the imidazolium tetrafluoroborate ILs with unsaturated side chains. $^{14}$

\section{Experimental Section}

${ }^{1} \mathrm{H}$ and ${ }^{13} \mathrm{C}$ NMR spectra were obtained using $\mathrm{CDCl}_{3}$ as solvent on a JEOL JNM LA-300 spectrometer (300 MHz for ${ }^{1} \mathrm{H}$ NMR and $75 \mathrm{MHz}$ for ${ }^{13} \mathrm{C}$ NMR). The ${ }^{1} \mathrm{H}$ NMR data were reported as follows in ppm $(\delta)$ from the internal standard (TMS, $0.0 \mathrm{ppm}$ ), chemical shift (multiplicity, coupling constant in $\mathrm{Hz}$, integration), and the ${ }^{13} \mathrm{C}$ NMR data in ppm $(\delta)$ from the internal standard (TMS, $0.0 \mathrm{ppm}$ ).

Thermal properties were analyzed by DSC and TGA. Crystallization point and melting point were determined by using TA Instruments Differential Scanning Calorimeter (DSC) Q1000 under $\mathrm{N}_{2}$ atmosphere. Thermograms were recorded during heating from $-80{ }^{\circ} \mathrm{C}$ to $100{ }^{\circ} \mathrm{C}$ scans at a heating rate of $10{ }^{\circ} \mathrm{C} \mathrm{min}{ }^{-1}$ after cooling to $-80{ }^{\circ} \mathrm{C}$ scans at a cooling rate of $10{ }^{\circ} \mathrm{C} \mathrm{min}^{-1}$. Thermal decomposition temperature was recorded by using TA Instruments SDT Q600 under Ar atmosphere. Heating rate and terminal temperature were set at $10{ }^{\circ} \mathrm{C} \mathrm{min}-1$ and $800{ }^{\circ} \mathrm{C}$, respectively. Density was measured by weighing a measured volume of the ILs.

Ionic conductivity was determined by the complex impedance measurements with platinum electrodes, using a CHI660A electrochemical workstation in the frequency ranges of 100 


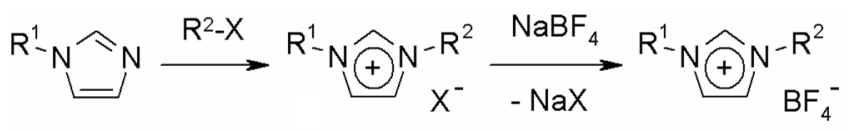

$$
\begin{aligned}
& \mathrm{R}^{1}=\text { methyl, vinyl, allyl } \\
& \mathrm{R}^{2}=\text { ethyl, propyl, butyl, isobutyl, } \\
& \text { propargyl, allyl, crotyl, methallyl } \\
& \mathrm{X}=\mathrm{Cl}, \mathrm{Br}
\end{aligned}
$$

Scheme 1. Preparation of the imidazolium tetrafluoroborates.

$\mathrm{kHz}$ and $1 \mathrm{~Hz}$. The cell constant of the cell was $1.6 \mathrm{~cm}^{-1}$, determined with 1.0 $\mathrm{M} \mathrm{LiPF}_{6} \mathrm{EC}: \mathrm{DMC}$ (vol. $1: 1$ ) electrolyte, where $\mathrm{EC}$ is ethylene carbonate and DMC is dimethyl carbonate. Cyclic voltammetry was performed with a CHI660A electrochemical workstation for the electrochemical stability window measurement at a scan rate of $10 \mathrm{mV} \mathrm{s}^{-1}$ by using platinum electrode $\left(1.96 \times 10^{-3} \mathrm{~cm}^{2}\right)$ as a working electrode. The Pt electrode was polished before every measurement. A platinum foil was used as a counter electrode and silver wire as a quasi-reference electrode. In this work, the cathodic and anodic limits were arbitrarily defined as the potential at which the current density reached $1 \mathrm{~mA} \mathrm{~cm}{ }^{-2}$. All the electrochemical measurements were made at room temperature unless otherwise specified.

Preparation of the ionic liquids. The imidazolium tetrafluoroborates with unsaturated aliphatic side chains were prepared according to the modified procedure described by Wilkes and Zaworotko (Scheme 1). ${ }^{4}$ Some imidazolium tetrafluoro- borates with saturated alkyl side chains were also made for comparison by using the same synthetic protocol.

General procedure for the preparation of the imidazolium halide. To a stirred solution of 1-methylimidazole (8.91 g, $100.0 \mathrm{mmol})$ in acetonitrile $(70 \mathrm{~mL})$ was added requisite aliphatic halide $\left(\mathrm{R}^{2}-\mathrm{X}, 110.0 \mathrm{mmol}\right)$ dropwise at $0{ }^{\circ} \mathrm{C}$. The reaction mixture was stirred for $24-48 \mathrm{~h}$ at $30{ }^{\circ} \mathrm{C}$. Removal of the solvent under reduced pressure afforded crude $1-\mathrm{R}^{2}-3$ methylimidazolium halide.

1-Allylimidazole instead of 1-methylimidazole was used for the preparation of 1-allyl-3-propargylimidazolium tetrafluoroborate $\left([\mathrm{PaAIm}] \mathrm{BF}_{4}\right)$, 1-allyl-3-crotylimidazolium tetrafluoroborate $\left([\mathrm{CAIm}] \mathrm{BF}_{4}\right)$ and 1-allyl-3-methallylimidazolium tetrafluoroborate ([MaAIm]BF 4 ). 1-Vinylimidazole was used as a starting imidazole for the preparation of 1-ethyl-3vinylimidazolium tetrafluoroborate $\left([\mathrm{VEIm}] \mathrm{BF}_{4}\right)$ and 1allyl-3-vinylimidazolium tetrafluoroborate $\left(\left[\mathrm{VAIm}_{\mathrm{B}} \mathrm{BF}_{4}\right)\right.$.

1,3-Diallylimidazolium bromide ([AAIm]Br) was prepared by modifying the procedure described by Guibé and coworkers as follows. ${ }^{15}$ To a stirred mixture of imidazole $(6.81 \mathrm{~g}, 100.0 \mathrm{mmol})$ and $\mathrm{NaHCO}_{3}(16.80 \mathrm{~g}, 200.0 \mathrm{mmol})$ in acetonitrile $(70 \mathrm{~mL})$ was added allyl bromide $(220.0 \mathrm{mmol})$ dropwise at $0{ }^{\circ} \mathrm{C}$. The reaction mixture was stirred for $24 \mathrm{~h}$ at room temperature. Filtration of the resulting mixture followed by removal of the solvent under reduced pressure afforded crude [AAIm]Br.

Completion of all the quaternization reaction was confirmed by ${ }^{1} \mathrm{H}$ and ${ }^{13} \mathrm{C}$ NMR. The crude product was used without further purification for the next step, the anion metathesis.

General procedure for the anion metathesis. To a solution of the crude imidazolium halide, obtained from each of the above reactions, in acetone $(70 \mathrm{~mL})$ was added sodium tetrafluoroborate $(10.90 \mathrm{~g}, 100.0 \mathrm{mmol})$. The reaction mixture was stirred for $24 \mathrm{~h}$ at room temperature. The resulting mixture was filtered through a pad of aluminum oxide to remove the sodium salt and color. Evaporation of the solvent under reduced pressure afforded the corresponding imidazolium tetrafluoroborate.

Completion of the reaction was confirmed by ${ }^{1} \mathrm{H}$ NMR. The $\mathrm{Na}$ content of all the imidazolium tetrafluoroborates prepared in the present study was less than $0.08 \mathrm{wt} \%$ by ICP and their water content was below $0.03 \mathrm{wt} \%$ by Karl-Fischer titration.

1-Ethyl-3-methylimidazolium tetrafluoroborate ([EMIm] BF $_{4}$ ) (1). [EMIm]Br: ${ }^{1} \mathrm{H}$ NMR $\delta 1.62$ (t, $\left.J=7.4,3 \mathrm{H}\right), 4.13$ (s, 3H), 4.42 (q, $J=7.4,2 \mathrm{H}), 7.44(\mathrm{~s}, 2 \mathrm{H}), 10.55(\mathrm{~s}, 1 \mathrm{H}) ;{ }^{13} \mathrm{C}$ NMR $\delta 15.0,35.9,44.4,121.5,123.1,135.8$.

[EMIm] $\mathrm{BF}_{4}$ : Yield 95\%; $T_{\mathrm{m}} 12{ }^{\circ} \mathrm{C} ; T_{\mathrm{d}} 450{ }^{\circ} \mathrm{C} ;{ }^{1} \mathrm{H}$ NMR $\delta$ $1.41(\mathrm{t}, J=7.3,3 \mathrm{H}), 3.83$ (s, 3H), 4.14 (q, $J=7.3,2 \mathrm{H}), 7.35$ (s, 1H), $7.41(\mathrm{~s}, 1 \mathrm{H}), 8.55(\mathrm{~s}, 1 \mathrm{H}) ;{ }^{13} \mathrm{C}$ NMR $\delta 15.2,36.0$, 45.1, 122.2, 123.8, 135.9 .

1-Methyl-3-propylimidazolium tetrafluoroborate ([PMIm] BF $_{4}$ ) (2). [PMIm]Br: ${ }^{1} \mathrm{H}$ NMR $\delta 0.92(\mathrm{t}, J=7.4,3 \mathrm{H}), 1.84-$ $1.96(\mathrm{~m}, 2 \mathrm{H}), 4.06(\mathrm{~s}, 3 \mathrm{H}), 4.24(\mathrm{t}, J=7.3,2 \mathrm{H}), 7.47(\mathrm{~s}, 1 \mathrm{H})$ $7.56(\mathrm{~s}, 1 \mathrm{H}), 10.29(\mathrm{~s}, 1 \mathrm{H}) ;{ }^{13} \mathrm{C} \mathrm{NMR} \delta 10.5,23.4,36.4$, 51.1, 122.1, 123.5, 136.7 .

[PMIm] $\mathrm{BF}_{4}$ : Yield 92\%; $T_{\mathrm{m}}-9{ }^{\circ} \mathrm{C} ; T_{\mathrm{d}} 450{ }^{\circ} \mathrm{C} ;{ }^{1} \mathrm{H}$ NMR $\delta$ $0.97(\mathrm{t}, J=7.3,3 \mathrm{H}), 1.85-1.97(\mathrm{~m}, 2 \mathrm{H}), 3.96(\mathrm{~s}, 3 \mathrm{H}), 4.15(\mathrm{t}$, $J=7.3,2 \mathrm{H}), 7.21(\mathrm{~s}, 1 \mathrm{H}), 7.24(\mathrm{~s}, 1 \mathrm{H}), 8.93(\mathrm{~s}, 1 \mathrm{H}) ;{ }^{13} \mathrm{C}$ NMR $\delta 10.6,23.5,36.2,51.4,122.7,123.9,136.1$.

1-Butyl-3-methylimidazolium tetrafluoroborate ([BMIm] BF $_{4}$ ) (3). [BMIm]Br: ${ }^{1} \mathrm{H}$ NMR $\delta 0.92(\mathrm{t}, J=7.3,3 \mathrm{H}), 1.28-$ $1.41(\mathrm{~m}, 2 \mathrm{H}), 1.82-1.92(\mathrm{~m}, 2 \mathrm{H}), 4.09(\mathrm{~s}, 3 \mathrm{H}), 4.29(\mathrm{t}, J=$ 7.3, 2H), 7.39 (s, 1H), $7.50(\mathrm{~s}, 1 \mathrm{H}), 10.43(\mathrm{~s}, 1 \mathrm{H}) ;{ }^{13} \mathrm{C}$ NMR $\delta 13.3,19.2,32.0,36.5,49.6,122.1,123.7,136.8$.

[BMIm] $\mathrm{BF}_{4}$ : Yield 96\%; $T_{\mathrm{d}} 450{ }^{\circ} \mathrm{C} ;{ }^{1} \mathrm{H}$ NMR $\delta 0.94(\mathrm{t}, J=$ 7.3, 3H), 1.29-1.42 (m, 2H), 1.80-1.90 (m, 2H), 3.95 (s, 3H), $4.17(\mathrm{t}, J=7.3,2 \mathrm{H}), 7.22(\mathrm{~s}, 1 \mathrm{H}), 7.26(\mathrm{~s}, 1 \mathrm{H}), 8.88(\mathrm{~s}, 1 \mathrm{H})$; ${ }^{13} \mathrm{C}$ NMR $\delta 13.4,19.6,32.1,36.2,49.8,122.6,123.9,136.1$.

1-Isobutyl-3-methylimidazolium tetrafluoroborate ([iBMIm] BF 4 ) (4). [iBMIm]Br: ${ }^{1} \mathrm{H}$ NMR $\delta 0.96(\mathrm{~d}, J=6.8,6 \mathrm{H}), 2.13-$ $2.31(\mathrm{~m}, 1 \mathrm{H}), 4.11(\mathrm{~s}, 3 \mathrm{H}), 4.12(\mathrm{~d}, J=7.3,2 \mathrm{H}), 7.31(\mathrm{~s}, 1 \mathrm{H})$, $7.45(\mathrm{~s}, 1 \mathrm{H}), 10.50(\mathrm{~s}, 1 \mathrm{H}) ;{ }^{13} \mathrm{C} \mathrm{NMR} \delta 19.6,29.7,36.9$, 57.0, 122.6, 123.7, 137.8 .

[iBMIm] $\mathrm{BF}_{4}$ : Yield $92 \% ; T_{\mathrm{d}} 430{ }^{\circ} \mathrm{C} ;{ }^{1} \mathrm{H}$ NMR $\delta 0.94(\mathrm{~d}, J$ $=6.6,6 \mathrm{H}), 2.08-2.18(\mathrm{~m}, 1 \mathrm{H}), 3.96(\mathrm{~s}, 3 \mathrm{H}), 3.99(\mathrm{~d}, J=7.5$, 2H), $7.21(\mathrm{~s}, 1 \mathrm{H}), 7.29(\mathrm{~s}, 1 \mathrm{H}), 8.85(\mathrm{~s}, 1 \mathrm{H}) ;{ }^{13} \mathrm{C} \mathrm{NMR} \delta$ 19.6, 29.6, 36.6, 57.3, 122.6, 123.7, 137.1.

1-Ethyl-3-vinylimidazolium tetrafluoroborate ([VEIm]BF 4$)$ (5). [VEIm]Br: ${ }^{1} \mathrm{H}$ NMR $\delta 1.63(\mathrm{t}, J=7.3,3 \mathrm{H}), 4.51(\mathrm{q}, J=$ $7.3,2 \mathrm{H}), 5.40(\mathrm{dd}, J=8.7$ and $3.1,1 \mathrm{H}), 5.95(\mathrm{dd}, J=15.7$ and $3.1,1 \mathrm{H}), 7.45(\mathrm{dd}, J=15.7$ and $8.7,1 \mathrm{H}), 7.50(\mathrm{~s}, 1 \mathrm{H})$, $7.68(\mathrm{~s}, 1 \mathrm{H}), 11.13(\mathrm{~s}, 1 \mathrm{H}) ;{ }^{13} \mathrm{C} \mathrm{NMR} \delta 15.8,45.9,110.1$, $119.5,122.7,128.4,136.0$.

[VEIm]BF 4 : Yield 82\%; $T_{\mathrm{c}}-54.1{ }^{\circ} \mathrm{C} ; T_{\mathrm{m}}-3.7{ }^{\circ} \mathrm{C} ; T_{\mathrm{d}} 384$ ${ }^{\circ} \mathrm{C}$; ${ }^{1} \mathrm{H}$ NMR $\delta 1.44(\mathrm{t}, J=7.2,3 \mathrm{H}), 4.21(\mathrm{q}, J=7.2,2 \mathrm{H})$, $5.28(\mathrm{dd}, J=8.8$ and $3.0,1 \mathrm{H}), 5.80(\mathrm{dd}, J=15.7$ and 3.0, 
$1 \mathrm{H}), 7.03(\mathrm{dd}, J=15.7$ and 8.8, 1H), $7.51(\mathrm{~s}, 1 \mathrm{H}), 7.71(\mathrm{~s}$, $1 \mathrm{H}), 8.85(\mathrm{~s}, 1 \mathrm{H}) ;{ }^{13} \mathrm{C}$ NMR $\delta 15.0,45.6,109.4,119.6$, $123.1,128.5,134.1$.

1-Methyl-3-propargylimidazolium tetrafluoroborate ([PaMIm]BF 4 ) (6). [PaMIm]Br: ${ }^{1} \mathrm{H}$ NMR $\delta 2.76(\mathrm{t}, J=2.6$, $1 \mathrm{H}), 4.09(\mathrm{~s}, 3 \mathrm{H}), 5.39(\mathrm{~d}, J=2.6,2 \mathrm{H}), 7.52(\mathrm{~s}, 1 \mathrm{H}), 7.63(\mathrm{~s}$, $1 \mathrm{H}), 10.39(\mathrm{~s}, 1 \mathrm{H}) ;{ }^{13} \mathrm{C}$ NMR $\delta 36.2,39.6,74.0,79.9,122.3$, 122.6, 135.8 .

[PaMIm] $\mathrm{BF}_{4}$ : Yield $85 \% ; T_{\mathrm{m}} 46.3{ }^{\circ} \mathrm{C} ; T_{\mathrm{d}} 363{ }^{\circ} \mathrm{C} ;{ }^{1} \mathrm{H}$ NMR $\delta 2.92(\mathrm{~s}, 1 \mathrm{H}), 3.79(\mathrm{~s}, 3 \mathrm{H}), 4.90$ (s, 2H), 7.29 (s, 1H), 7.41 $(\mathrm{s}, 1 \mathrm{H}), 8.54(\mathrm{~s}, 1 \mathrm{H}) ;{ }^{13} \mathrm{C}$ NMR $\delta 36.2,39.5,73.8,77.9$, $122.2,122.4,135.5$.

1-Allyl-3-methylimidazolium tetrafluoroborate ([AMIm] BF $_{4}$ ) (7). [AMIm]Br: ${ }^{1} \mathrm{H}$ NMR $\delta 4.08(\mathrm{~s}, 3 \mathrm{H}), 4.97$ (d, $J=$ $6.4,2 \mathrm{H}), 5.42(\mathrm{~d}, J=10.1,1 \mathrm{H}), 5.45(\mathrm{~d}, J=17.0,1 \mathrm{H}), 5.91-$ $6.05(\mathrm{~m}, 1 \mathrm{H}), 7.39(\mathrm{~s}, 1 \mathrm{H}), 7.55(\mathrm{~s}, 1 \mathrm{H}), 10.33(\mathrm{~s}, 1 \mathrm{H}) ;{ }^{13} \mathrm{C}$ NMR $\delta 36.7,51.6,122.0,122.1,123.8,130.2,136.4$.

[AMIm] $\mathrm{BF}_{4}$ : Yield $98 \% ; T_{\mathrm{d}} 400{ }^{\circ} \mathrm{C} ;{ }^{1} \mathrm{H}$ NMR $\delta 3.96(\mathrm{~s}$, $3 \mathrm{H}), 4.79(\mathrm{~d}, J=6.6,2 \mathrm{H}), 5.46(\mathrm{~d}, J=17.2,1 \mathrm{H}), 5.47(\mathrm{~d}, J=$ 10.1, 1H), 5.92-6.05 (m, 1H), 7.21 (s, 1H), 7.25 (s, 1H), 8.87 (s, $1 \mathrm{H}) ;{ }^{13} \mathrm{C}$ NMR $\delta 36.4,51.5,121.7,121.8,123.6,129.9$, 136.1 .

1-Crotyl-3-methylimidazolium tetrafluoroborate ([CMIm] BF $\left._{4}\right)$ (8). [CMIm]Cl: ${ }^{1} \mathrm{H}$ NMR $\delta 1.74(\mathrm{~d}, J=6.6,3 \mathrm{H}), 4.08$ (s, 3H), $4.86(\mathrm{~d}, J=6.8,2 \mathrm{H}), 5.55-5.65(\mathrm{~m}, 1 \mathrm{H}), 5.92-6.03$ (m, 1H), $7.26(\mathrm{~s}, 1 \mathrm{H}), 7.41(\mathrm{~s}, 1 \mathrm{H}), 10.72(\mathrm{~s}, 1 \mathrm{H}) ;{ }^{13} \mathrm{C}$ NMR $\delta 18.0,36.8,51.8,121.5,122.7,123.6,135.5,138.1$.

[CMIm] $\mathrm{BF}_{4}$ : Yield $88 \% ; T_{\mathrm{d}} 348{ }^{\circ} \mathrm{C} ;{ }^{1} \mathrm{H}$ NMR $\delta 1.75(\mathrm{~d}, J$ $=6.6,3 \mathrm{H}), 4.09(\mathrm{~s}, 3 \mathrm{H}), 4.68(\mathrm{~d}, J=7.0,2 \mathrm{H}), 5.56-5.66(\mathrm{~m}$, $1 \mathrm{H}), 5.92-6.04(\mathrm{~m}, 1 \mathrm{H}), 7.24(\mathrm{~s}, 1 \mathrm{H}), 7.31(\mathrm{~s}, 1 \mathrm{H}), 8.73(\mathrm{~s}$, $1 \mathrm{H}) ;{ }^{13} \mathrm{C}$ NMR $\delta 17.8,36.3,51.8,121.6,122.2,123.5,130.8$, 135.9.

1-Methallyl-3-methylimidazolium tetrafluoroborate ([MaMIm]BF 4 ) (9). [MaMIm]Cl: ${ }^{1} \mathrm{H}$ NMR $\delta 1.73$ (s, 3H), $4.11(\mathrm{~s}, 3 \mathrm{H}), 4.91(\mathrm{~s}, 2 \mathrm{H}), 4.98(\mathrm{~s}, 1 \mathrm{H}), 5.12(\mathrm{~s}, 1 \mathrm{H}), 7.16(\mathrm{~s}$, $1 \mathrm{H}), 7.26(\mathrm{~s}, 1 \mathrm{H}), 11.10(\mathrm{~s}, 1 \mathrm{H}) ;{ }^{13} \mathrm{C}$ NMR $\delta 19.5,36.3,55.3$, 116.9, 122.8, 124.2, 136.5, 138.4.

[MaMIm] $\mathrm{BF}_{4}$ : Yield $90 \% ; T_{\mathrm{d}} 400{ }^{\circ} \mathrm{C} ;{ }^{1} \mathrm{H}$ NMR $\delta 1.71(\mathrm{~s}$, $3 \mathrm{H}), 3.96(\mathrm{~s}, 3 \mathrm{H}), 4.70(\mathrm{~s}, 2 \mathrm{H}), 5.00(\mathrm{~s}, 1 \mathrm{H}), 5.11(\mathrm{~s}, 1 \mathrm{H})$, $7.21(\mathrm{~s}, 1 \mathrm{H}), 7.33(\mathrm{~s}, 1 \mathrm{H}), 8.85(\mathrm{~s}, 1 \mathrm{H}) ;{ }^{13} \mathrm{C} \mathrm{NMR} \delta 19.5$, $36.4,55.1,116.8,121.7,123.9,137.6,137.7$.

1-Allyl-3-vinylimidazolium tetrafluoroborate ([VAIm] $\left.\mathbf{B F}_{4}\right)$ (10). [VAIm]Br: ${ }^{1} \mathrm{H}$ NMR $\delta 5.07$ (d, $\left.J=6.6,2 \mathrm{H}\right), 5.39$ (dd, $J$ $=8.6$ and $3.1,1 \mathrm{H}), 5.47(\mathrm{~d}, J=9.9,1 \mathrm{H}), 5.51(\mathrm{~d}, J=16.7$, $1 \mathrm{H}), 5.94-6.09(\mathrm{~m}, 2 \mathrm{H}), 7.42(\mathrm{dd}, J=15.6$ and $8.6,1 \mathrm{H}), 7.48$ $(\mathrm{s}, 1 \mathrm{H}), 7.80(\mathrm{~s}, 1 \mathrm{H}), 10.96(\mathrm{~s}, 1 \mathrm{H}) ;{ }^{13} \mathrm{C}$ NMR $\delta 52.5,110.3$, 119.6, 122.6, 123.5, 128.4, 129.6, 136.2.

[VAIm]BF 4 : Yield $89 \% ; T_{\mathrm{c}}-25.1{ }^{\circ} \mathrm{C} ; T_{\mathrm{m}} 53.2{ }^{\circ} \mathrm{C} ; T_{\mathrm{d}} 330$ ${ }^{\circ} \mathrm{C} ;{ }^{1} \mathrm{H}$ NMR $\delta 4.86(\mathrm{~d}, J=6.6,2 \mathrm{H}), 5.42(\mathrm{dd}, J=8.7$ and $3.1,1 \mathrm{H}), 5.48-5.52(\mathrm{~m}, 2 \mathrm{H}), 5.72(\mathrm{dd}, J=15.6$ and $3.1,1 \mathrm{H})$, 5.93-6.07 (m, 1H), $7.13(\mathrm{dd}, J=15.6$ and 8.7, 1H), $7.33(\mathrm{~s}$, $1 \mathrm{H}), 7.54(\mathrm{~s}, 1 \mathrm{H}), 9.20(\mathrm{~s}, 1 \mathrm{H}) ;{ }^{13} \mathrm{C}$ NMR $\delta 52.5,110.2$, $119.4,122.6,123.4,128.0,129.2,136.0$.

1-Allyl-3-propargylimidazolium tetrafluoroborate ([PaAIm] BF $_{4}$ ) (11). $[\mathrm{PaAIm}] \mathrm{Br}:{ }^{1} \mathrm{H}$ NMR $\delta 2.71(\mathrm{t}, J=2.6,1 \mathrm{H}), 4.99$ $(\mathrm{d}, J=6.4,2 \mathrm{H}), 5.42(\mathrm{~d}, J=2.6,2 \mathrm{H}), 5.48(\mathrm{~d}, J=10.6,1 \mathrm{H})$, $5.49(\mathrm{~d}, J=16.2,1 \mathrm{H}), 5.94-6.08(\mathrm{~m}, 1 \mathrm{H}), 7.37(\mathrm{~s}, 1 \mathrm{H}), 7.61$ (s, 1H), 10.55 (s, 1H); ${ }^{13} \mathrm{C}$ NMR $\delta 39.7,52.0,74.0,78.1$, 122.2, 122.4, 122.6, 129.6, 135.9.

$[\mathrm{PaAIm}] \mathrm{BF}$ : : Yield $71 \% ; T_{\mathrm{c}}-65.0{ }^{\circ} \mathrm{C} ; T_{\mathrm{m}}-18.0^{\circ} \mathrm{C} ; T_{\mathrm{d}} 342$ ${ }^{\circ} \mathrm{C} ;{ }^{1} \mathrm{H}$ NMR $\delta 2.68(\mathrm{t}, J=2.4,1 \mathrm{H}), 4.82(\mathrm{~d}, J=6.4,2 \mathrm{H})$, $5.10(\mathrm{~d}, J=2.4,2 \mathrm{H}), 5.51(\mathrm{~d}, J=18.2,1 \mathrm{H}), 5.52(\mathrm{~d}, J=9.7$, 1H), 5.92-6.07 (m, 1H), $7.24(\mathrm{~s}, 1 \mathrm{H}), 7.47(\mathrm{~s}, 1 \mathrm{H}), 9.10(\mathrm{~s}$, $1 \mathrm{H}) ;{ }^{13} \mathrm{C}$ NMR $\delta 39.5,51.7,73.9,77.8,122.0,122.2,122.5$, 129.3, 135.7 .

1,3-Diallylimidazolium tetrafluoroborate ([AAIm] $\mathbf{B F}_{4}$ ) (12). [AAIm]Br: ${ }^{1} \mathrm{H}$ NMR $\delta 5.01$ (d, $\left.J=6.4,4 \mathrm{H}\right), 5.44$ (dd, $J$ $=10.1$ and $0.7,2 \mathrm{H}), 5.47(\mathrm{dd}, J=16.8$ and $0.7,2 \mathrm{H}), 5.94-$ $6.07(\mathrm{~m}, 2 \mathrm{H}), 7.40(\mathrm{~s}, 2 \mathrm{H}), 10.49(\mathrm{~s}, 1 \mathrm{H}) ;{ }^{13} \mathrm{C}$ NMR $\delta 52.1$, $122.3,122.7,129.9,136.6$.

[AAIm] $\mathrm{BF}_{4}$ : Yield $93 \% ; T_{\mathrm{c}}-52.8{ }^{\circ} \mathrm{C} ; T_{\mathrm{m}} 4.3{ }^{\circ} \mathrm{C} ; T_{\mathrm{d}} 380$ ${ }^{\circ} \mathrm{C} ;{ }^{1} \mathrm{H}$ NMR $\delta 4.79(\mathrm{~d}, J=6.6,4 \mathrm{H}), 5.44(\mathrm{~d}, J=9.9,2 \mathrm{H})$, $5.45(\mathrm{~d}, J=17.6,2 \mathrm{H}), 5.92-6.05(\mathrm{~m}, 2 \mathrm{H}), 7.29(\mathrm{~s}, 2 \mathrm{H}), 8.86$ (s, $1 \mathrm{H}) ;{ }^{13} \mathrm{C}$ NMR $\delta 52.4,122.3,123.1,129.8,136.3$.

1-Allyl-3-crotylimidazolium tetrafluoroborate ([CAIm] $\left.\mathbf{B F}_{4}\right)$ (13). [CAIm]Cl: ${ }^{1} \mathrm{H}$ NMR $\delta 1.74(\mathrm{~d}, J=6.6,3 \mathrm{H}), 4.89(\mathrm{~d}, J$ $=6.9,2 \mathrm{H}), 5.01(\mathrm{~d}, J=6.6,2 \mathrm{H}), 5.42(\mathrm{~d}, J=10.5,1 \mathrm{H}), 5.43$ $(\mathrm{d}, J=16.8,1 \mathrm{H}), 5.56-5.66(\mathrm{~m}, 1 \mathrm{H}), 5.91-6.05(\mathrm{~m}, 2 \mathrm{H}), 7.19$ $(\mathrm{s}, 1 \mathrm{H}), 7.29(\mathrm{~s}, 1 \mathrm{H}), 10.80(\mathrm{~s}, 1 \mathrm{H}) ;{ }^{13} \mathrm{C}$ NMR $\delta 17.7,51.5$, 51.8, 121.8, 122.1, 122.2, 122.6, 130.0, 135.1, 136.7.

[CAIm]BF 4 : Yield $97 \% ; T_{\mathrm{c}}-57.7^{\circ} \mathrm{C} ; T_{\mathrm{m}}-15.4{ }^{\circ} \mathrm{C} ; T_{\mathrm{d}} 345$ ${ }^{\circ} \mathrm{C} ;{ }^{1} \mathrm{H}$ NMR $\delta 1.75(\mathrm{~d}, J=6.6,3 \mathrm{H}), 4.70(\mathrm{~d}, J=7.0,2 \mathrm{H})$, 4.78 (d, $J=6.6,2 \mathrm{H}), 5.45$ (d, $J=17.8,1 \mathrm{H}), 5.46$ (d, $J=11.2$, $1 \mathrm{H}), 5.57-5.67(\mathrm{~m}, 1 \mathrm{H}), 5.91-6.04(\mathrm{~m}, 2 \mathrm{H}), 7.28(\mathrm{~s}, 2 \mathrm{H})$, $8.80(\mathrm{~s}, 1 \mathrm{H}) ;{ }^{13} \mathrm{C}$ NMR $\delta 17.5,51.3,51.6,120.9,121.6$, $121.7,122.7,129.8,134.7,136.2$.

1-Allyl-3-methallylimidazolium tetrafluoroborate ([MaAIm] BF $\left._{4}\right)$ (14). [MaAIm]Cl: ${ }^{1} \mathrm{H}$ NMR $\delta 1.72(\mathrm{~s}, 3 \mathrm{H}), 4.93(\mathrm{~s}$, $2 \mathrm{H}), 4.97(\mathrm{~s}, 1 \mathrm{H}), 5.03(\mathrm{~d}, J=6.4,2 \mathrm{H}), 5.10(\mathrm{~s}, 1 \mathrm{H}), 5.44(\mathrm{~d}$, $J=17.0,1 \mathrm{H}), 5.45(\mathrm{~d}, J=9.7,1 \mathrm{H}), 5.93-6.06(\mathrm{~m}, 1 \mathrm{H}), 7.23$ (s, 1H), $7.31(\mathrm{~s}, 1 \mathrm{H}), 11.03(\mathrm{~s}, 1 \mathrm{H}) ;{ }^{13} \mathrm{C}$ NMR $\delta$ 19.3, 51.6, 55.1, 116.7, 121.9, 122.1, 122.2, 129.7, 137.2, 138.6.

[MaAIm]BF 4 : Yield $92 \% ; T_{\mathrm{d}} 355{ }^{\circ} \mathrm{C} ;{ }^{1} \mathrm{H}$ NMR $\delta 1.70(\mathrm{~s}$, 3H), $4.72(\mathrm{~s}, 2 \mathrm{H}), 4.81(\mathrm{~d}, J=6.6,2 \mathrm{H}), 5.00(\mathrm{~s}, 1 \mathrm{H}), 5.10(\mathrm{~s}$, $1 \mathrm{H}), 5.44(\mathrm{~d}, J=10.8,1 \mathrm{H}), 5.46(\mathrm{~d}, J=15.9,1 \mathrm{H}), 5.92-6.06$ $(\mathrm{m}, 1 \mathrm{H}), 7.26(\mathrm{~s}, 1 \mathrm{H}), 7.32(\mathrm{~s}, 1 \mathrm{H}), 8.86(\mathrm{~s}, 1 \mathrm{H}) ;{ }^{13} \mathrm{C} \mathrm{NMR} \delta$ 19.6, 51.8, 55.3, 116.9, 122.1, 122.2, 122.4, 130.0, 137.5, 137.8 .

\section{Results and Discussion}

Thermal behaviors. Table 1 lists the crystallization point $\left(T_{\mathrm{c}}\right)$, melting point $\left(T_{\mathrm{m}}\right)$ and decomposition temperature $\left(T_{\mathrm{d}}\right)$ of the ILs prepared in this work and some selected DSC charts are shown in Figure 1. The thermal properties of ILs with the saturated side chains are similar to those reported in the literature. ${ }^{3,6}$ Most of the ILs with unsaturated side chains are liquid at room temperature. Several ILs such as $[\mathrm{VEIm}] \mathrm{BF}_{4}$, $[$ VAIm $] \mathrm{BF}_{4},\left[\mathrm{PaAIm}_{\mathrm{B}} \mathrm{BF}_{4},[\mathrm{AAIm}] \mathrm{BF}_{4}\right.$, and $[\mathrm{CAIm}] \mathrm{BF}_{4}$ show $T_{\mathrm{c}}$ and $T_{\mathrm{m}}$ (entries 5, 10,11, 12 and 13).

The existence of $T_{\mathrm{c}}$ and $T_{\mathrm{m}}$ seems to be resulted from the symmetry and planarity of the compounds. The [AAIm] $\mathrm{BF}_{4}$ molecules would pack each other effectively because of the symmetric nature. Therefore, it was easy to find the melting 
Table 1. The structures and physicochemical properties of the ionic liquids prepared

\begin{tabular}{|c|c|c|c|c|c|c|c|c|c|}
\hline No. & Ionic liquids & $\mathrm{R}^{1}$ & $\mathrm{R}^{2}$ & $T_{\mathrm{c}}\left({ }^{\circ} \mathrm{C}\right)$ & $T_{\mathrm{m}}\left({ }^{\circ} \mathrm{C}\right)$ & $T_{\mathrm{d}}\left({ }^{\circ} \mathrm{C}\right)$ & $\mathrm{d}(\mathrm{g} / \mathrm{mL})$ & $\eta(\mathrm{cP})$ & $\sigma(\mathrm{mS} / \mathrm{cm})$ \\
\hline 1 & {$\left[\mathrm{EMIm}_{\mathrm{B} F}\right.$} & Ethyl & Methyl & $\mathrm{ND}^{a}$ & 12 & 450 & 1.28 & 27 & 14.4 \\
\hline 2 & {$[\mathrm{PMIm}] \mathrm{BF}_{4}$} & Propyl & Methyl & ND & -9 & 450 & 1.24 & 58 & 7.2 \\
\hline 3 & 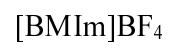 & Butyl & Methyl & ND & ND & 450 & 1.17 & 76 & 4.3 \\
\hline 4 & {$[\mathrm{iBMIm}] \mathrm{BF}_{4}$} & Isobutyl & Methyl & ND & ND & 430 & 1.15 & 76 & 1.8 \\
\hline 5 & {$[\mathrm{VEIm}] \mathrm{BF}_{4}$} & Ethyl & Vinyl & -54 & -4 & 384 & $1.25^{b}$ & $41^{b}$ & $10.5^{b}$ \\
\hline 6 & {$[\mathrm{PaMIm}] \mathrm{BF}_{4}$} & Propargyl & Methyl & ND & 46 & 363 & $1.29^{b}$ & $47^{b}$ & $7.0^{b}$ \\
\hline 7 & {$\left[\mathrm{AMIm} \mathrm{BF}_{4}\right.$} & Allyl & Methyl & ND & ND & 400 & 1.33 & 45 & 10.0 \\
\hline 8 & {$[\mathrm{CMIm}] \mathrm{BF}_{4}$} & Crotyl & Methyl & ND & ND & 348 & 1.22 & 63 & 6.2 \\
\hline 9 & {$[\mathrm{MaMIm}] \mathrm{BF}_{4}$} & Methallyl & Methyl & ND & ND & 400 & 1.29 & 136 & 2.4 \\
\hline 10 & {$[\mathrm{VAIm}] \mathrm{BF}_{4}$} & Allyl & Vinyl & -25 & 53 & 330 & $1.22^{b}$ & $29^{b}$ & $15.0^{c}$ \\
\hline 11 & {$[\mathrm{PaAIm}] \mathrm{BF}_{4}$} & Propargyl & Allyl & -65 & -18 & 342 & 1.24 & 222 & 2.5 \\
\hline 12 & {$[\mathrm{AAIm}] \mathrm{BF}_{4}$} & Allyl & Allyl & -53 & 4 & 380 & 1.22 & 48 & 8.6 \\
\hline 13 & {$[\mathrm{CAIm}] \mathrm{BF}_{4}$} & Crotyl & Allyl & -58 & -15 & 345 & 1.16 & 57 & 5.3 \\
\hline 14 & {$[\mathrm{MaAIm}] \mathrm{BF}_{4}$} & Methallyl & Allyl & ND & ND & 355 & 1.14 & 96 & 4.0 \\
\hline
\end{tabular}

${ }^{a}$ ND: not detected. ${ }^{b}$ Measured at $50{ }^{\circ} \mathrm{C} .{ }^{c}$ Measured at $60{ }^{\circ} \mathrm{C}$



Figure 1. The DSC charts of some selected ionic liquids.

point and the crystallization point of [AAIm] $\mathrm{BF}_{4}$ (entry 12). This tendency could be found in $\left[\mathrm{PaAIm}_{\mathrm{B}} \mathrm{BF}_{4}\right.$ and $[\mathrm{CAIm}] \mathrm{BF}_{4}$ that have the nearly linear and planar structures (entries 11 and 13). The intermolecular interaction would be enhanced by the fully conjugated and planar structure caused by the vinyl group in $[\mathrm{VEIm}] \mathrm{BF}_{4}$ and $\left[\mathrm{VAIm}_{\mathrm{B}} \mathrm{BF}_{4}\right.$ (entries 5 and
10). ${ }^{16}$ Other cations are relatively unsymmetrical owing to the different sizes of the substituents so that their $T_{m}$ are hardly observed. It has been also reported that the melting points for the imidazolium cations containing a longer alkyl chain were not observed. ${ }^{6 c}$

All of the imidazolium tetrafluoroborates with the unsaturated side chains are thermally less stable than the corresponding ILs with saturated side chains that are stable up to $430{ }^{\circ} \mathrm{C}$. The ILs with one unsaturated side chain are a little more stable than those with two unsaturated side chains. However, they are all thermally much more stable compared with conventional carbonate organic electrolytes.

Physical properties. Table 1 also shows density (d), viscosity $(\eta)$ and ionic conductivity $(\sigma)$ of the ILs prepared in this work. Generally, ionic conductivity is proportional to the number of charge carrier ions and their mobility. It is well known that larger cations make the ILs more viscous because of the increased intermolecular van der Waals interactions. ${ }^{1,3,6}$ That is, any longer and bulkier side chain in imidazolium cation reduces the rotational freedom of molecules, which leads to a higher viscosity and lower ionic conductivity. This feature is observed in Table 1 for all of the imidazolium salts with saturated or unsaturated side chains. However, the imidazolium salts with an allyl or crotyl side chain show lower viscosities and better conductivities compared with their corresponding saturated analogues (entries 7 and 12 vs. 2, entries 8 and 13 vs. 3). In particular,

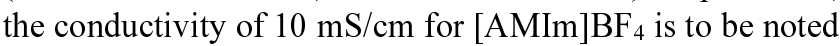
because this number is large enough to be considered as a battery or capacitor electrolyte (entry 7).

The higher conductivity of the imidazolium salts with the unsaturated side chains could be explained in terms of their molecular structures. A more planar structure is known to be less viscous because the planarity allows relatively facile 
slip between molecules. ${ }^{17}$ In this work, the relatively planar allyl or crotyl group can be more parallel to the imidazolium ring and seems to hold the planarity of the cation compared to the saturated alkyl substituents. Branching of the methyl group in the methallyl group would distort the planarity because of the steric hindrance between the methyl substituent of the methallyl group and imidazole ring, resulting in a higher viscosity and lower conductivity (entries 7 and $8 v s$. 9, entries 12 and 13 vs. 14).

Electrochemical behavior. The electrochemical stability windows of the ILs determined from their cyclic voltammograms are listed in Table 2 and typical cyclic voltammograms are represented in Figure 2. The cathodic limits in Table 2 are similar for all of the ILs examined. Interestingly, however, the anodic limits are dependent on the nature of alkyl substituent on the imidazolium ring. The ILs with

Table 2. Electrochemical windows of the ionic liquids prepared

\begin{tabular}{|c|c|c|c|c|}
\hline No. & Ionic liquids & $\begin{array}{l}\text { Cathodic limi } \\
\left(\mathrm{V}, v s . \mathrm{Ag} / \mathrm{Ag}^{+}\right.\end{array}$ & $\begin{array}{l}\text { Anodic limit } \\
\left(\mathrm{V}, v s \cdot \mathrm{Ag} / \mathrm{Ag}^{+}\right)\end{array}$ & $\begin{array}{l}\text { Electrochemica } \\
\text { window }(V)\end{array}$ \\
\hline 1 & {$[\mathrm{EMIm}] \mathrm{BF}_{4}$} & -1.6 & 2.5 & 4.1 \\
\hline 2 & {$\left[\mathrm{PMIm}_{\mathrm{B} F}\right.$} & -1.7 & 2.4 & 4.1 \\
\hline 3 & {$[\mathrm{BMIm}] \mathrm{BF}_{4}$} & -1.8 & 2.5 & 4.3 \\
\hline 4 & {$[\mathrm{iBMIm}] \mathrm{BF}_{4}$} & -1.8 & 2.2 & 4.0 \\
\hline 5 & {$\left[\right.$ VEIm] $\mathrm{BF}_{4}{ }^{a}$} & -1.3 & 2.7 & 4.0 \\
\hline 6 & {$[\mathrm{PaMIm}] \mathrm{BF}_{4}{ }^{b}$} & -1.5 & 4.2 & 5.7 \\
\hline 7 & {$[\mathrm{AMIm}] \mathrm{BF}_{4}$} & -1.4 & 4.0 & 5.4 \\
\hline 8 & {$[\mathrm{CMIm}] \mathrm{BF}_{4}$} & -1.7 & 3.6 & 5.3 \\
\hline 9 & {$[\mathrm{MaMIm}] \mathrm{BF}_{4}$} & -1.8 & 4.2 & 6.0 \\
\hline 10 & {$\left[\mathrm{VAIm} \mathrm{BF}_{4}{ }^{a}\right.$} & -1.2 & 2.4 & 3.6 \\
\hline 11 & {$[\mathrm{PaAIm}] \mathrm{BF}_{4}{ }^{b}$} & -1.7 & 5.5 & 7.2 \\
\hline 12 & {$[\mathrm{AAIm}] \mathrm{BF}_{4}$} & -1.6 & 4.2 & 5.8 \\
\hline 13 & {$[\mathrm{CAIm}] \mathrm{BF}_{4}$} & -1.6 & 4.3 & 5.9 \\
\hline 14 & {$[\mathrm{MaAIm}] \mathrm{BF}_{4}$} & -1.6 & 4.0 & 5.6 \\
\hline
\end{tabular}

${ }^{a}$ Measured at $60{ }^{\circ} \mathrm{C} .{ }^{b}$ Supercooled liquid.

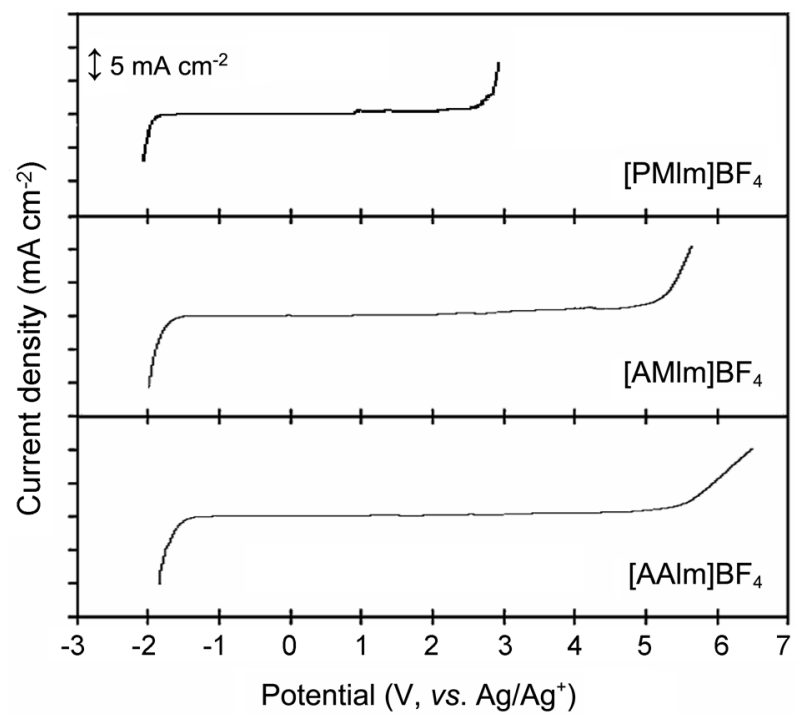

Figure 2. Cyclic voltammograms of the allyl-substituted imidazolium tetrafluoroborates. saturated side chains exhibit an anodic limit of $<2.5 \mathrm{~V}$ ( $v s$. $\mathrm{Ag} / \mathrm{Ag}^{+}$) (entries 1-4), ${ }^{3,6}$ which is contrasted by the higher anodic limit shown by the ILs with unsaturated side chains (entries 5-14).

Usually, both the cathodic and anodic limits would be determined by decomposition of the cations and the anions, respectively. The cathodic decomposition is resulted from the reduction of the acidic proton located at the $\mathrm{C}-2$ position of the imidazolium ring, whereas the anodic decomposition is deeply associated with the stability of anions on highly positively polarized electrode surface. ${ }^{1 a, 6 a}$ The observation, where the ILs with unsaturated side chains show a higher anodic limit than that of the saturated counterparts even if they carry the same $\mathrm{BF}_{4}{ }^{-}$anion, manifests itself that the side chains on the imidazolium ring play an important role in affecting the anodic limit. It is proposed that a certain type of passivation layer is developed on the Pt electrode as a result of oxidation of unsaturated side chains. Note that the double bond is more vulnerable to electrochemical oxidation than the saturated one. If this is the case, the passivation layer would suppress the decomposition of the $\mathrm{BF}_{4}{ }^{-}$anion to extend the anodic limit. An easier formation of passivation layer with unsaturated molecules has been reported for allyl ethyl carbonate, vinylene carbonate, and vinyl ethylene carbonate that are frequently used as an electrolyte additive in lithium secondary batteries. These unsaturated molecules are oxidatively decomposed to form a passivation layer on cathode surface so as to suppress the further decomposition of organic electrolytes. ${ }^{18}$ In this sense, it is very likely that the ILs having the unsaturated side chains, with their wide electrochemical stability windows, would be useful for their applications as an electrolyte in electrochemical capacitors and lithium secondary batteries. Preliminary results made for battery application are, however, still unsatisfactory as reported by others. ${ }^{10}$ The performance improvement by a proper selection of additive is now under study.

\section{Conclusions}

The imidazolium tetrafluoroborates with the unsaturated aliphatic side chains were synthesized, and their physicochemical properties were compared with those of the ILs containing the saturated side chains. Most of the ILs with the unsaturated side chains are liquid at room temperature. All of them are thermally less stable than the saturated analogues but more stable than common organic carbonate electrolytes. Some imidazolium tetrafluoroborates with an allylic side chain showed wider electrochemical stability windows on Pt electrode, better conductivities, and lower viscosities compared with the corresponding ILs containing the saturated side chains. The higher ionic conductivity shown by them is attributed to the lower viscosity that is rationalized by their relatively planar structures. The remarkably high anodic limits shown by them in the cyclic voltammograms is explained by the surface passivation that is resulted from an electrochemical oxidation of unsaturated side chains such as the allylic group. 
Acknowledgement. This work was supported by the Division of Advanced Batteries in NGE Program (Project No. 10016439) and by KOSEF through the Research Center for Energy Conversion and Storage.

\section{References}

1. (a) Bonhôte, P.; Dias, A.-P.; Armand, M.; Papageorgiou, N.; Kalyanasundaram, K.; Grätzel, M. Inorg. Chem. 1996, 35, 11681178. (b) Buzzeo, M. C.; Evans, R. G.; Compton, R. G. ChemPhysChem 2004, 5, 1106-1120. (c) Lee, J. S.; Bae, J. Y.; Lee, H.; Quan, N. D.; Kim, H. S.; Kim, H. J. Ind. Eng. Chem. 2004, 10, 1086-1089.

2. For reviews, see: (a) Welton, T. Chem. Rev. 1999, 99, 2071-2083. (b) Song, C. E.; Yoon, M. Y.; Choi, D. S. Bull. Korean Chem. Soc. 2005, 26, 1321-1330. (c) Jorapur, Y. R.; Chi, D. Y. Bull. Korean Chem. Soc. 2006, 27, 345-354.

3. Anthony, J. L.; Brennecke, J. F.; Holbrey, J. D.; Maginn, E. J.; Mantz, R. A.; Rogers, R. D.; Trulove, P. C.; Visser, A. E.; Welton, T. In Ionic Liquids in Synthesis; Wasserscheid, P.; Welton, T., Eds.; Wiley-VCH Verlag: Weinheim, 2003; pp 41-126.

4. Wilkes, J. S.; Zaworotko, M. J. J. Chem. Soc., Chem. Commun. 1992, 965-967.

5. (a) Webber, A.; Blomgren, G. E. In Advances in Lithium-Ion Batteries; van Schalkwijk, W. A.; Scrosati, B., Eds.; Kluwer Academic/Plenum Publishers: New York, 2002; pp 185-232. (b) In Electrochemical Aspects of Ionic Liquids; Ohno, H., ed.; WileyInterscience: Hoboken, 2005; pp 173-223.

6. (a) Hagiwara, R.; Ito, Y. J. Fluorine Chem. 2000, 105, 221-227. (b) Nishida, T.; Tashiro, Y.; Yamamoto, M. J. Fluorine Chem. 2003, 120, 135-141. (c) Holbrey, J. D.; Seddon, K. R. J. Chem. Soc., Dalton Trans. 1999, 2133-2139.

7. (a) Ue, M.; Takeda, M.; Takahashi, T.; Takehara, M. Electrochem. Solid State Lett. 2002, 5, A119-A121. (b) Balducci, A.; Bardi, U.; Caporali, S.; Mastragostino, M.; Soavi, F. Electrochem. Commun.
2004, 6, 566-570. (c) Sato, T.; Masuda, G.; Takagi, K. Electrochim. Acta 2004, 49, 3603-3611.

8. Wang, P.; Zakeeruddin, S. M.; Exnar, I.; Grätzel, M. Chem. Commun. 2002, 2972-2973.

9. de Souza, R. F.; Padilha, J. C.; Gonçalves, R. S.; Dupont, J. Electrochem. Commun. 2003, 5, 728-731.

10. Garcia, B.; Lavallée, S.; Perron, G.; Michot, C.; Armand, M. Electrochim. Acta 2004, 49, 4583-4588.

11. (a) Zhou, Z.-B.; Matsumoto, H.; Tatsumi, K. Chem. Eur. J. 2004, 10, 6581-6591. (b) Yoshida, Y.; Muroi, K.; Otsuka, A.; Saito, G.; Takahashi, M.; Yoko, T. Inorg. Chem. 2004, 43, 1458-1462.

12. (a) Visser, A. E.; Swatloski, R. P.; Reichert, W. M.; Mayton, R.; Sheff, S.; Wierzbicki, A.; Davis, J. H., Jr.; Rogers, R. D. Chem. Commun. 2001, 135-136. (b) Zhao, D.; Fei, Z.; Scopelliti, R.; Dyson, P. J. Inorg. Chem. 2004, 43, 2197-2205.

13. Presented in part at the 31 st Korean Society of Industrial and Engineering Chemistry National Meeting, Daejeon, Korea, May 13-14, 2005, No. 1P-178.

14. The ILs of allyl-substituted imidazolium with halide anions have been reported recently; (a) Mizumo, T.; Marwanta, E.; Matsumi, N.; Ohno, H. Chem. Lett. 2004, 33, 1360-1361; (b) Kölle, P.; Dronskowski, R. Eur. J. Inorg. Chem. 2004, 2313-2320. The synthesis and characterization of two allyl-substituted imidazoliums with the $\mathrm{BF}_{4}{ }^{-}$anion have been reported; (c) Zhao, D.; Fei, Z.; Geldbach, T. J.; Scopelliti, R.; Laurenczy, G.; Dyson, P. J. Helv. Chim. Acta 2005, 88, 665-675.

15. Kimbonguila, A. M.; Boucida, S.; Guibé, F.; Loffet, A. Tetrahedron 1997, 53, 12525-12538.

16. Similar differences in melting points can be found in the following compounds with the comparable structures; propylbenzene $(\mathrm{mp}$ $\left.-99{ }^{\circ} \mathrm{C}\right)$ vs allylbenzene $\left(\mathrm{mp}-40{ }^{\circ} \mathrm{C}\right)$ and ethylbenzene $(\mathrm{mp}-95$ $\left.{ }^{\circ} \mathrm{C}\right)$ vs styrene $\left(\mathrm{mp}-31{ }^{\circ} \mathrm{C}\right)$.

17. Sun, J.; MacFarlane, D. R.; Forsyth, M. Electrochim. Acta 2003, $48,1707-1711$.

18. Lee, J.-T.; Lin, Y.-W.; Jan, Y.-S. J. Power Sources 2004, 132, $244-$ 248. 